\title{
Retrofuturismo en tiempos de Covid-19: cómo las predicciones catastrofistas del pasado nos ayudan en el presente
}

\author{
Retrofuturism in the time of Covid-19: how the \\ catastrophic predictions of the past help us \\ in the present
}

\author{
José-Pablo Gallo-León
}

Gallo-León, José-Pablo (2019). "Retrofuturismo en tiempos de Covid-19: cómo las predicciones catastrofistas del pasado nos ayudan en el presente". Anuario ThinkEPI, v. 14, e14b02.

https://doi.org/10.3145/thinkepi.2020.e14b02

Publicado en IweTel el 24 de junio de 2020

\section{José-Pablo Gallo-León}

https://orcid.org/0000-0002-8236-4275

Universidad de Alicante. Biblioteca.

Universidad de Murcia. Departamento de Información

y Documentación

jpablo.gallo@ua.es

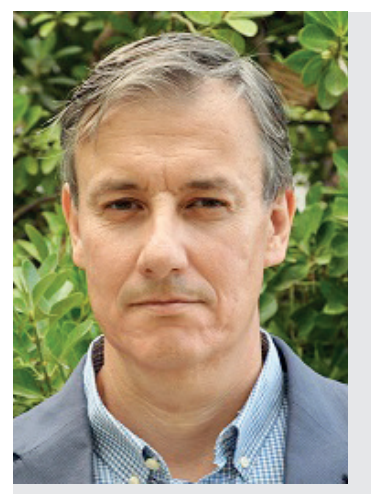

Resumen: El cierre de los servicios presenciales de las bibliotecas con motivo de la pandemia por la Covid-19 ha provocado que muchas voces recuerden la necesidad de mejorar los servicios digitales. Sin embargo, este entorno digital ha sido visto durante mucho tiempo como una amenaza para la existencia de las propias bibliotecas. Las visiones más negativas al respecto no se cumplieron, pero probablemente nos prepararon para evolucionar hacia nuevos modelos de biblioteca. Esta mayor presencia de lo digital no contradice la importancia que sigue teniendo la biblioteca como lugar.

Palabras clave: Bibliotecas digitales; Biblioteca como lugar; Covid-19; Futuro de las bibliotecas.

Abstract: The closure of library locations due to the Covid-19 pandemic has resultvoices restating the need to improve digital services. However, this digital environment has long been seen as a threat to the existence of libraries themselves. The accuracy of the worst predictions is questionable, but those visions probably prepared us to evolve towards new library models. These models, with a greater presence of digital, do not contradict the importance of the library as a place.

Keywords: Digital libraries; Library as a place; Covid-19; Future of libraries.

\section{Introducción}

Recientemente, múltiples voces se han elevado para solicitar que las bibliotecas profundicemos en la digitalización a raíz del confinamiento. Sin embargo, la digitalización masiva ha sido argumentada de forma repetida como amenaza para la existencia de la biblioteca en tiempos pasados. Estas visiones catastrofistas, básicamente incumplidas, quizá hayan resultado positivas.

Obviamente, entendemos digitalización no como el mero hecho de pasar las colecciones a soportes digitales, sino como una concepción digital de los servicios bibliotecarios. 
La visión de un futuro oscuro puede ser una buena herramienta para tener un futuro brillante o, al menos, llevadero. No vamos a entrar en los mecanismos psicológicos de anticipación negativa, ni en el juego de si era posible anticiparse a la pandemia. Tampoco se va a realizar un artículo sobre el steampunk, o movimientos estéticos similares, pero sí vamos a echar un vistazo a lo que se decía no hace mucho del futuro de la biblioteca. Recuperaremos ejemplos de grandes profesionales que realizaron predicciones muy negativas sobre la influencia de la tecnología digital en las bibliotecas. Ninguna se ha cumplido plenamente. Incluso alguna de ellas, en absoluto. Personalmente, siempre se ha sido muy crítico, incluso desdeñoso, con esa actitud, pero este texto es casi una retractación, pues se empieza a entender su valor. Pensar futuros oscuros para las bibliotecas lleva 50 años siendo una costumbre y, quizá, no ha ido tan mal.

La visión negativa es una herramienta que se utiliza dentro de la metodología de escenarios, bien es cierto que junto con otras predicciones más positivas: se imaginan posibles escenarios en los que nos podemos encontrar en un futuro para anticipar una respuesta. Esto ha sido trabajado muy bien por algunos estudios de futuro, como The bookends scenarios (State Library of New South Wales, 2009).

Estos estudios sobre el futuro de las bibliotecas y sus profesionales, ya lo hemos dicho otras veces, pueden considerarse una plaga en sí mismos. Lluís Anglada (2019) afirmaba que se publicaron más de 500 artículos sobre el futuro de las bibliotecas entre 2011 y 2016. Personalmente se ha tenido noticia de 35 estudios desde 2008 sobre la cuestión, vinculados a grandes instituciones (asociaciones, organizaciones sin ánimo de lucro, entidades públicas). Es lógico, dada la incertidumbre que ha generado el entorno digital y sus consecuencias directas sobre una profesión que se basa en el manejo de la información. Hoy, con la Covid-19 aún presente, esta inquietud se ha acentuado y muchos se replantean el papel de la biblioteca en una etapa en que la presencialidad física ha sido imposible.

\section{Intensificar la digitalización tras el confinamiento}

Las voces que están proponiendo que profundicemos en la digitalización de nuestro entorno son variadas y de prestigio. Por poner dos ejemplos destacados:

- Recientemente, las 13 bibliotecas del CSUC publicaron un manifiesto sobre

"la necesidad de acelerar la transformación digital de la educación superior, que se ha hecho aún más evidente a causa de la crisis sanitaria provocada por la Covid-19" (CSUC, 2020).

- Por otra parte, ha habido muchos artículos sobre Covid y bibliotecas, pero claro, no todos aparecen en The New York Times ni están firmados por el presidente de la Biblioteca Pública de Nueva York ${ }^{1}$. Bajo el contundente título "Las bibliotecas deben cambiar", Anthony W. Marx indica que, a raíz de la Covid, es evidente que hay que apostar por lo digital. Para permanecer fieles a su misión durante la pandemia, las bibliotecas deberían ofrecer más servicios digitales (Marx, 2020).

En la práctica, estas posturas responden a una realidad que hemos experimentado los bibliotecarios: ante el cierre de nuestras instalaciones, el uso de las colecciones digitales se ha incrementado. Incluso servicios a los que les había costado coger la velocidad de crucero, como eBiblio, han visto como subían sus estadísticas, llegando la noticia a la prensa².

Estos llamamientos suponen un aldabonazo para que se produzcan unos cambios que, pese a lo que solemos afirmar, no se habían consumado plenamente. Las bibliotecas nos hemos ido adaptando a las realidades cambiantes, pero quizá no tanto como nos gusta presumir. No hay más que ver la cantidad de bibliotecas públicas que no tienen, siquiera, página web ${ }^{3}$. Y en las universitarias, Natalia Arroyo y José Antonio Gómez nos acaban de enseñar la deficiente integración que tienen las bibliotecas académicas españolas en las plataformas y campus virtuales de sus instituciones (Arroyo-Vázquez; Gómez-Hernández, 2020).

\section{La visión catastrofista sobre la digitalización}

Sin embargo, la digitalización ha sido vista, y en realidad lo sigue siendo para la sociedad, como la gran amenaza del mundo bibliotecario, que iba a provocar su extinción. Es cierto que son visiones reduccionistas de la biblioteca, que se centran en el binomio edificio-libro como única realidad, pero no dejan de tener su lógica a primera vista.

El ejemplo más notable de autor que vaticinó el fin de las bibliotecas físicas y su imparable cambio a

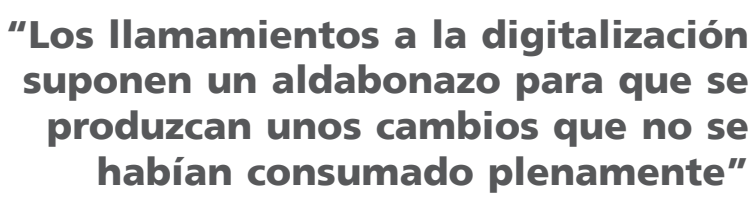


lo digital fue F. W. Lancaster, sobre todo en una serie de trabajos publicados entre finales de los años 70 y principios de los 80 del siglo XX (Lancaster, 1978a; 1978b; 1982; 1983). El autor predecía su sustitución por bibliotecas digitales, bastante antes de la www, y anticipaba la necesidad de que los bibliotecarios tuviésemos los conocimientos necesarios para ello.

Carlson (2001) publicó un artículo en la Chronicle of higher education que iba a tener un gran impacto: "The deserted library". Reflejó, suscitando un gran debate en la profesión, el temor a que la biblioteca se vaciase debido al acceso electrónico a la documentación.

Esto se reproduce, una década más tarde, en el no menos famoso artículo de Coffman (2012), según el cual las bibliotecas vivíamos bajo la amenaza de nuestra redundancia frente a fuentes de información alternativas.

Un año antes, y curiosamente (o no), también en la Chronicle of Higher Education, Sullivan (2011) publicó un breve artículo, "Academic library autopsy report, 2050", de inequívoco título. Mostraba en cinco puntos su idea de que las bibliotecas se convertirían en insostenibles por coste/beneficio, pues la consulta de información y, esto es aún más doloroso, la formación en su manejo, serían más sencillas y rápidas por otros medios ${ }^{4}$.

Hay acercamientos más humorísticos, como el del post de Aaron Tay, The day library discovery died, que podemos ver traducido al español por el Grupo Durga (2013). En él ironizaba sobre la futilidad de los intentos de crear herramientas de descubrimiento que compitiesen con los grandes buscadores, mientras que los bibliotecarios iban a ser sustituidos por robots. Sin embargo, 7 años después no se imagina una biblioteca universitaria con un sistema de gestión que no integre una buena herramienta de descubrimiento.

Incluso, en un extremo de desprecio hacia la necesidad de las bibliotecas, merced a esa visión restringida, hay académicos que afirman que no son más que un gasto inútil, como cuando Adrian Sannier, de la Arizona State University, dijo: "Stop air conditioning the books" (Fister, 2009).

\section{Lo digital no ha sido el fin}

¿Qué se ha conseguido con todas estas agoreras predicciones? Por una parte, volver a evidenciar la dificultad de hacerlas, aún desde el punto de vista de profesionales expertos, conocedores en profundidad de las tendencias. Pero aportan mucho más que eso: anunciándonos lo peor, nos han preparado

“Lo digital no es una vía excluyente. El espacio físico seguirá jugando un importante papel en nuestros centros"

para intentar ir a caballo de ese entorno digital. Sin la visión de Lancaster, quizá nos habríamos adaptado finalmente a la automatización, primero, y al entorno red, después, pero seguramente nos habría costado mucho más. Son señales de alarma que nos ayudan a movernos. No importa que no se cumplan, porque muchos de sus autores ni siquiera parecen desearlo, sino que expresan unos temores íntimos, como Coffman, Carlson o Tay. De ahí la gran utilidad que tienen y han tenido para nuestro desarrollo.

No nos engañemos, seguiremos recibiendo la pregunta sobre la necesidad de la biblioteca y observaciones, si se permite, tan cafres como la de Sannier por muchos años. Y de esos miedos vendrán acicates e impulsos para adaptarnos y cambiar. Podríamos hablar de darwinismo, pero es que las bibliotecas llevan adaptándose a las tecnologías desde su origen, pasando por todos los soportes, medios y condiciones espaciales imaginables desde Mesopotamia hasta ahora.

A raíz de ello, es interesante recuperar la viñeta (figura 1) del ilustrador Chris OBrion de 2009, adaptada por el Grupo Durga (2015) bajo el título "¿Seguimos necesitando bibliotecas?" Como decían en su post, "¿por qué llevamos desde 2009 haciendo esta pregunta?" Es que llevamos mucho más. Y lo que nos queda.

\section{Pequeña reivindicación del espacio}

Hemos hablado de digitalización, pero es importante decir que, siendo esta fundamental, no excluye el espacio físico.

El propio artículo en The New York Times también dice que

"sería irresponsable, y me atrevo a decir que peligroso, proclamar que la biblioteca del futuro está aquí, y es solo en línea. Incluso en un mundo digital, las bibliotecas físicas juegan un papel fundamental en nuestras comunidades" (Marx, 2020).

Se habla de la capacidad de acogimiento, de dar un servicio gratuito a todos, de poder interactuar con los semejantes. En definitiva, del importante rol social de las bibliotecas públicas.

Este importante papel de la biblioteca como espacio social, llámese tercer lugar o lo que queramos, es particularmente importante para las minorías. Echando mano, como ya es habitual en la bibliografía profesional, de los estudios del Pew Research Center, por ejemplo, se decía que el $42 \%$ de los usuarios de 


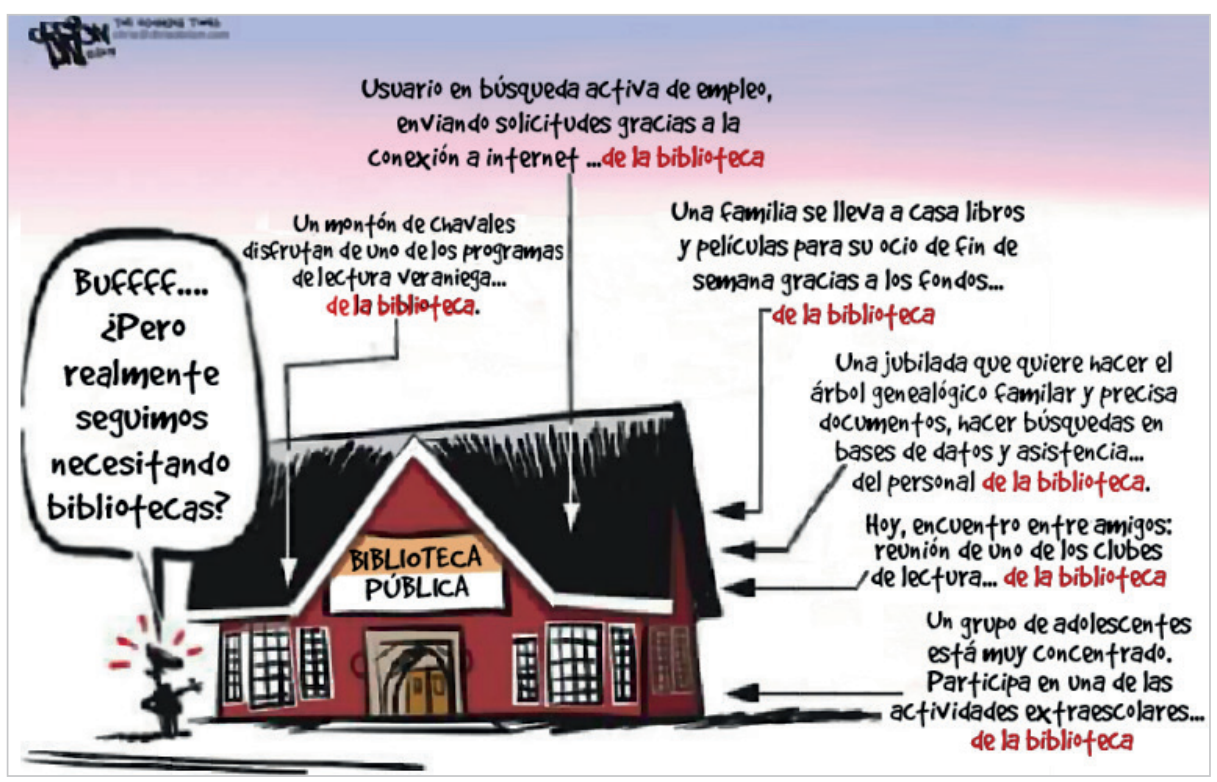

Figura 1. ¿Seguimos necesitando bibliotecas? Viñeta de Chris OBrion ${ }^{5}$ (Grupo Durga, 2015)

biblioteca afroamericanos utilizaban los ordenadores y la conexión a internet de las bibliotecas públicas. Esto, frente al $25 \%$ de los blancos y, sorprendentemente, el $24 \%$ de los hispanos, que no dejan de ser cifras muy altas. Evidencian el valor que pueden tener los servicios de biblioteca física en un entorno digital. Además, otro estudio de este centro señalaba a los millennials como la generación más proclive al uso de las bibliotecas públicas, reforzando por tanto su vigencia (Geiger, 2017).

De una forma más cercana, Funes reivindica el espacio físico de la biblioteca y su bibliotecidad, su cualidad intrínseca como tal. Al tiempo, también nos recuerda que este ha de ser reinventado. Que se necesita que los bibliotecarios nos despertemos y hagamos por fin cambios:

"Si se acomete la reducción de espacio para el estudio que sea con la finalidad de abordar, por fin, ese $f a b l a b$; ese espacio multidisciplinar; ese vivero de empresas; esas salas para reuniones/trabajos de grupo; ese estudio de grabación; o cualquiera otra reestructuración de espacios/servicios que, hasta ahora, no se había encontrado el momento de acometer" (Funes, 2020).

\section{Conclusiones}

En definitiva, esta crisis de lo presencial puede ser el impulso definitivo para que las bibliotecas nos adaptemos a lo digital de forma completa. Esto, después de que tantas predicciones agoreras durante casi cincuenta años nos advirtieran de que era la vía de su siguiente transformación y podía ser el único camino para evitar que fueran relegadas. Pero no nos engañemos, no es una vía excluyente. El espacio físico seguirá jugando un importante papel en nuestros centros.

\section{Notas}

1. Aunque no venga a cuento, no es bibliotecario, y en los órganos directivos de la NYPL solo aparece uno: https://www.nypl.org/help/about-nypl/president-and-leadership

\section{Por ejemplo:}

https://diariodecastillayleon.elmundo.es/articulo/castilla-y-leon/p/ataformas-digitales-ebiblio-cinecyl-sumanusuarios-servicios-confinamiento/20200405134333006689.html

https://www.lavanguardia.com/vida/20200510/481073221847/el-prestamo-de-libros-por-ebiblio-aumenta-en-abrilun-152--respecto-a-2019. html

3. Aunque sea dentro de una muestra muy limitada y de centros relativamente pequeños, en una reciente encuesta sobre comunicación en bibliotecas en la Región de Murcia se encontró que un $40 \%$ de las mismas no tenían página web (Gallo-León; Quílez-Simón, 2020).

4. Lili Li escribió dos obras sucesivas sobre el futuro muy orientadas a las tecnologías y en torno a este artículo, pero sobre todo la primera (Li, 2013), prácticamente dedicada a refutar las afirmaciones de Sullivan (2011).

5. La caricatura de Chris Obrion se creó para The Roanoke Times en 2009, pero se volvió viral en 2015, cuando Library journal la publicó en Facebook. La viñeta está disponible en múltiples sitios en internet, pero no es accesible en su fuente original porque tienen cerrado el acceso a la Unión Europea, debido a la Regulación de Protección de Datos. Igualmente, la página web del autor está inactiva y no recuperable plenamente en el WayBack Machine. 


\section{Referencias}

Anglada, Lluís (2019). "Tendencias futuras para las bibliotecas universitarias en un entorno de información digital, abierta y en la red". En: XVII Conferencia Internacional sobre Bibliotecas Universitarias (23 al 25 de octubre, 2019) [Inédito]. Ciudad de México: UNAM.

Arroyo-Vázquez, Natalia; Gómez-Hernández, José-Antonio (2020). “La biblioteca integrada en la enseñanza universitaria online: situación en España". Profesional de la información, v. 29, n. 4, e290404. https://doi.org/10.3145/epi.2020.jul.04

Carlson, Scott (2001). "The deserted library". The chronicle of higher education, v. 48, n. 12, pp. A35-A38. https://www.researchgate.net/publication/234695651_The_Deserted_Library

Coffman, Steve (2012). "The decline and fall of the librarian empire". Information today, v. 20, n. 3. http://www.infotoday.com/searcherlapr12/Coffman--The-Decline-and-Fall-of-the-Library-Empire.shtml

CSUC (2020). Manifiesto de las bibliotecas universitarias ante la Covid-19, 22 junio. https://www.csuc.cat/es/novedad/manifiesto-de-las-bibliotecas-universitarias-ante-la-covid-19

Fister, Barbara (2009). "The glorious study hall: how libraries nurture a life of the mind". Library issues: Briefings for faculty and administrators, v. 3, n. 2.

http://homepages.gac.edu/ fister/LibrarylssuesNov09.pdf

Funes, Vicente (2020). "Vindicación de lo bibliotecario en tiempos posCovid”. Infobibliotecas, 4 junio. https://blog.infobibliotecas.com/vindicacion-de-lo-bibliotecario-en-tiempos-poscovid/vicente-funes/

Gallo-León, José-Pablo; Quílez-Simón, Pedro (2020). “La biblioteca pública como comunicadora: procedimientos, canales y dificultades. El ejemplo de la Región de Murcia (España)". Anales de Documentación, v. 23, n. 2. https://revistas.um.es/analesdoc/article/view/403411 https://doi.org/10.6018/analesdoc.403411

Geiger. A. W. (2017). "Millennials are the most likely generation of Americans to use public libraries". Pew Research Center. Fact Tank, June 21.

https://www.pewresearch.org/fact-tank/2017/06/21/millennia/s-are-the-most-likely-generation-of-americans-to-use-public-libraries/

Grupo Durga (2013). "La encontraron muerta". Bibliotecas 2029, 11 de noviembre. https://bibliotecas2029.wordpress.com/2013/11/11/discovery-died

Grupo Durga (2015). "¿Seguimos necesitando bibliotecas?" Bibliotecas 2029, 4 septiembre. https://bibliotecas2029.wordpress.com/2015/09/04/seguimos-necesitando-bibliotecas-i

Lancaster, Frederick W. (1978a). Toward paperless information systems. New York: Academic Press. ISBN: 9780 124360501

Lancaster, Frederick W. (1978b). "Whither libraries? or, wither libraries". College and research libraries, v. 39, n. 5, pp. 345-357.

https://doi.org/10.5860/crl_39_05_345

Lancaster, Frederick W. (1982). Libraries and librarians in an age of electronics. Arlington: Information Resources Press. ISBN: 9780878150403

Lancaster, Frederick W. (1983). "Future librarianship: preparing for an unconventional career". Wilson library bulletin, v. 57, n. 9, pp. 747-753.

Li, Lili (2013). "The future of academic libraries in the digital age". En: Baker, David; Evans, Wendy (eds.). Trends, discovery, and people in the digital age, pp. 253-268. ISBN: Chandos. ISBN: 9781843347231 https://doi.org/10.1016/B978-1-84334-723-1.50016-4

Marx, Anthony W. (2020). "Libraries must change". The New York Times. Opinion, May 28. https://www.nytimes.com/2020/05/28/opinion/libraries-coronavirus.html

Perrin, Andrew; Turner, Erica (2019). "Smartphones help blacks, Hispanics bridge some - but not all - digital gaps with whites". Pew Research Center. Fact Tank, August 20.

https://www.pewresearch.org/fact-tank/2019/08/20/smartphones-help-blacks-hispanics-bridge-some-but-not-alldigital-gaps-with-whites/

State Library of NSW (2009). The bookends scenarios: Alternative futures for the Public Library Network in NSW in 2030. Sydney: State Library of NSW Management Records. ISBN: 073137200 X https://www.sl.nsw.gov.au/sites/default/files/bookends_scenarios.pdf

Sullivan, Brian T. (2011). "Academic library autopsy report, 2050". The chronicle of higher education, January 02. https://www.chronicle.com/article/Academic-Library-Autopsy/125767 